\title{
Diversity, equity, and access in psychedelic medicine
}

\author{
MONNICA T. WILLIAMS ${ }^{1,2} *$ and BEATRIZ C. LABATE ${ }^{3,4}$ \\ ${ }^{1}$ School of Psychology, University of Ottawa, Ottawa, Canada \\ ${ }^{2}$ Department of Psychology, University of Connecticut, Storrs, CT, USA \\ ${ }^{3}$ Chacruna Institute for Psychedelic Plant Medicine, San Francisco, CA, USA \\ ${ }^{4}$ East-West Psychology Program, California Institute for Integral Studies, San Francisco, CA, USA
}

(Received: October 29, 2019; accepted: November 12, 2019)

\begin{abstract}
Although it is exciting to witness the culmination of decades of drug policy advocacy and clinical research, the psychedelic science movement struggles with many of the same social issues that plague healthcare in general. The healing properties of plant medicines and their derivatives were originally brought to Western consciousness by indigenous cultures from all over the world. These practices are now being adapted to Western models of healthcare, in part, to achieve governmental approval as medical treatments. The current models of psychedelic psychotherapy being utilized in clinical trials are resource-intensive and therefore likely to remain out of reach for the socioeconomically disadvantaged if approved as medical treatments. Moreover, people of color and women are uncommon in leadership positions in the psychedelic research community, and few people of color are included as research participants in psychedelic studies. This piece introduces a special issue with a focus on issues of diversity, equity, and accessibility in psychedelic medicine.
\end{abstract}

Keywords: psychedelics, diversity, race, culture, therapy

With the advancement of psychedelic science into the forefront of global consciousness, research continues to affirm the promise of psychedelic medicines to aid in the healing of those suffering from trauma, addiction, depression, anxietyrelated disorders, and a host of other conditions. Several top universities have started their own centers for psychedelic research - including Johns Hopkins University and the University of Toronto - and educational programs are now being developed to train clinicians to deliver psychedelicassisted therapies (Phelps, 2017). Yet, despite these developments, and an impressive spate of progressive and well-intentioned international investigators, the science as a whole remains severely lacking in diversity and inclusion.

The healing properties of plant medicines and their derivatives were originally brought to Western consciousness by indigenous cultures around the world. These practices must now be adapted to Western models of healthcare to promote access and achieve governmental approval as medical treatments. The current models of psychedelic psychotherapy being utilized in clinical trials are resourceintensive and therefore likely to remain out of reach for the socioeconomically disadvantaged once approved as medical treatments. Moreover, people of color are uncommon in leadership positions in the psychedelic research community, and few are included as research participants in psychedelic studies (e.g., Michaels, Purdon, Collins, \& Williams, 2018). This special issue is focused on these issues of diversity, equity, and accessibility in psychedelic medicine. We are pleased to include nine excellent articles, from anthropology to clinical psychology, by authors from diverse backgrounds, including Black, Asian, Hispanic, indigenous, immigrant, and queer contributors.
The first article in our issue, by George, Michaels, Sevelius, and Williams (2019), is focused on what has been termed the "psychedelic renaissance" and problems imposed by the prevailing White-dominant medical framework, calling for greater indigenous, female, and ethnic minority inclusion in the field as a whole. The scientific progress and the clinical promise of psychedelic medicine owes much of its success to the history of indigenous healing practices, the work of people of color, and other disenfranchised groups, but this goes largely unacknowledged in the mainstream narrative of psychedelic medicine (e.g., Pollan, 2018). George et al. (2019) address this issue by highlighting the traditional role of psychedelic plants, summarizing the history of psychedelic medicine, and then exploring the social factors that have limited inclusion or acknowledgment of certain groups for their contributions. Finally, the article provides recommendations for broadening the Western medical framework of healing to include a cultural focus and considerations for an inclusive approach to treatment development and dissemination for future studies.

Following this piece, Fotiou (2019) explores the role of indigenous knowledge in psychedelic science. Although the beginnings of psychedelic science were steeped in colonialism, the study of psychedelics has helped us to better understand indigenous knowledge systems. Recent calls to

\footnotetext{
* Corresponding author: Monnica T. Williams, PhD; School of Psychology, University of Ottawa, 136 Jean-Jacques Lussier, Vanier Hall, Ottawa, ON K1N 6N5, Canada; Phone: +1 613 562 5801; Mobile: +1 343961 1290; Fax: +1 613562 5169; E-mail: Monnica.Williams@uOttawa.ca
}

This is an open-access article distributed under the terms of the Creative Commons Attribution-NonCommercial 4.0 International License, which permits unrestricted use, distribution, and reproduction in any medium for non-commercial purposes, provided the original author and source are credited, a link to the CC License is provided, and changes - if any - are indicated. 
decolonize theoretical frameworks and methodology open space for engaging more meaningfully with indigenous worldviews. At this critical juncture of the psychedelic renaissance, it is important to reflect on shortcomings of the current model and how we might genuinely restructure psychedelic science. The author calls for a much-needed shift in paradigm, a revision of the biomedical model that places indigenous worldviews on an equal plane with scientific inquiry to make Western biomedical models more inclusive. She asserts that decolonizing psychedelic science will require allowing multiple perspectives to coexist and contribute equally to efforts moving forward.

There is much to be learned from indigenous cultures, yet it is important to understand that traditional healers are human beings the same as everyone else, and while most are dedicated to helping and serving others, some might have more ambiguous intentions. With rising interest in the healing properties of the plant medicine, ayahuasca, people from all over the world have been increasingly traveling to the Amazon to partake in traditional ceremonies with Amazonian shamans; many shamans also now travel to the West for this purpose. Peluso Sinclair, Labate, and Cavnar (2019) describe an unfortunate associated and growing risk, as some participants in ayahuasca ceremonies have been sexually assaulted by individuals conducting or facilitating those ceremonies. The authors raise the key issues and processes that have led to formation, publication, and dissemination of the Chacruna Institute for Psychedelic Plant Medicines' Ayahuasca Community Guide for the Awareness of Sexual Abuse, focusing specifically on the needs for such guidelines as well as the challenges faced in collaboratively creating them. The authors also stress the importance of research and experience in understanding the complexities of the contexts in which potential abuse can occur, particularly around consent and intercultural communication. The overall aim is to educate at all levels by better informing ayahuasca participants and to be part of a broader goal of creating awareness around issues of sexual abuse.

Noorani (2019) tackles the politics and paradoxes of medicalization of psychedelics. This commentary draws on ethnographic research from 2014 to date, together with examples from psychiatry and drug research, to explore the issue within in wider political, economic, and cultural contexts. He argues that the resurgence of psychedelic science, which has been concerned with medicalization from the outset, has caused a crisis in the psychedelic community over its self-identity and values. Attempts to develop and market psychedelic-assisted therapy could end up undermining the safety and efficacy of the therapy itself. He also argues that psychedelic medicalization may have the unintended effect of hindering decriminalization and legalization efforts by delineation of licit versus illicit use of these substances.

Williams, Reed, and Aggarwal (2019) describe culturally informed research design and treatment issues, with respect to a 3,4-methylenedioxy-methamphetamine (MDMA)assisted psychotherapy study for post-traumatic stress disorder (PTSD) at a university medical center, sponsored by the Multidisciplinary Association for Psychedelic Studies (MAPS). This effort was notable due to its deliberate focus on the provision of culturally informed mental healthcare to people of color. The authors describe the development of their study site focused on the ethnic minority trauma experience, which included the clinical assessment of racial trauma, redesign of informed consent documents to improve understanding and acceptability, diversification of the treatment team, and ongoing diversity training for team members. The development of this site also included an examination of the setting and music used during sessions for cultural congruence, training and supervision for the external independent rater pool, and engagement with local communities of color. There is much to be learned from the authors' challenging experiences for diversifying future studies and training therapists to better serve clients of color.

The article also mentioned an upcoming MAPSsponsored conference, which has since occurred in Louisville, Kentucky, in August (Lekhtman, 2019). The conference launched with a successful opening workshop entitled, "Psychedelic Medicine and Cultural Trauma," followed by a 6.5 -day training for qualified therapists. This was the first event of its kind, where over 50 people of color began a training program to deliver MDMA psychotherapy to people suffering from PTSD (Carlin, in press). One sobering fact that made this event particularly salient is the legacy of research abuses that occurred in Kentucky, where scores of incarcerated Black men were used as experimental subjects in early unsafe and unethical psychedelic research trials at the Addiction Research Center at the Public Health Service Hospital in Lexington (e.g., Rosenberg, Wolbach, Miner, \& Isbell, 1963).

Neitzke-Spruill's (2019) article extends the topic of culturally informed psychedelic therapies by delving into an examination of race as a component of set and setting in the therapy experience. He notes that indigenous cultures around the world have been mindful of these factors for centuries, yet the concept of set and setting was not popularized until the mid-20th century, with little theoretical development among psychedelic researchers since that time. By bridging set and setting theory with research from the fields of social psychology and sociology of medicine, the article calls attention to how race becomes part of the set and setting during the psychedelic experience. He suggests that the problematic nature of race relations in the United States provides a distinct cultural setting for psychedelic users of color, and furthermore, psychedelic experiences may also reflect experiences of racialization. These considerations have important implications for developing protocols for the clinical applications of psychedelics, educating future practitioners, and meeting the needs of diverse patient populations. In addition, greater recognition and study of the role of race in relation to set and setting may enhance our understanding of non-pharmacological factors on psychedelic experiences.

Ching (2019) describes the salience of race, sexuality, and other cultural themes in his own experience receiving MDMA-assisted psychotherapy as a training exercise for his work as a psychedelic therapist for the study described by Williams, Reed, et al. (2019). The author provides a unique perspective, contrasting harsh drug laws his home country of Singapore against his legitimized (legal) involvement in the therapist training trials for MDMA-assisted psychotherapy for PTSD in the United States. Drawing upon clinical 
observations and personal insights in these trials, he discusses the importance of this work and gives a personal perspective on why he continues to participate in related academic and therapeutic service roles, despite cultural stigmas.

Finally, Eriacho (2019) shares her experience and wisdom for psychedelic therapists working with Native Americans. Therapists and other practitioners who hope to engage with Native groups should work toward understanding the cultural fundamentals common to Native Americans as a whole. Although individual and group differences certainly exist, critical areas of awareness include trust, respect, confidentiality, and inclusion of Native American traditions and culture in treatment. Although no one from the outside can ever perfectly understand a new culture, the very act of making efforts to understand Native culture goes a long way toward building rapport.

As emphasized by our notable contributors, psychedelic medicine as a movement struggles from similar social issues plaguing other fields of healthcare, resulting in disparities in inclusion and limiting our understanding of how to best help many groups that comprise our multicultural societies (Williams, Rosen, \& Kanter, 2019). However, there are several individuals and groups working hard to bring about positive change. In addition to the work by MAPS to train clinicians of color, we would like to outline the important work being done through the Chacruna Institute for Psychedelic Plant Medicines, lending talents to amplifying the voices of people of color in psychedelics (Chacruna's Racial Equity and Access Committee, 2019). We also thank our contributors, guest editorial board members, and reviewers for their contributions that have brought this special issue to fruition. Their voices and vision give us hope as they illuminate a path for the field to achieve the diversity, equity, and access to psychedelic healing that is so urgently needed. May this be just the beginning of a larger discussion that opens doors and heralds many rich contributions to come.

Conflict of interest: The authors declare no conflict of interest.

\section{REFERENCES}

Carlin, S. (in press). MDMA therapy training for communities of color. MAPS Bulletin.

Chacruna's Racial Equity and Access Committee. (2019, October 18). People of color making a difference in psychedelic healing. Chacruna Institute for Psychedelic Plant Medicines. Retrieved from https://chacruna.net/people-of-color-making-adifference-in-psychedelic-healing/
Ching, T. H. W. (2019). Intersectional insights from an MDMAassisted psychotherapy training trial: An open letter to racial/ ethnic and sexual/gender minorities. Journal of Psychedelic Studies. Advance online publication. doi:10.1556/2054. 2019.017

Eriacho, B. (2019). Considerations for psychedelic therapists when working with Native American people and communities. Journal of Psychedelic Studies. Advance online publication. doi:10.1556/2054.2019.030

Fotiou, E. (2019). The role of indigenous knowledges in psychedelic science. Journal of Psychedelic Studies. Advance online publication. doi:10.1556/2054.2019.031

George, J. R., Michaels, T. I., Sevelius, J., \& Williams, M. T. (2019). The psychedelic renaissance and the limitations of a White-dominant medical framework: A call for indigenous and ethnic minority inclusion. Journal of Psychedelic Studies. Advance online publication. doi:10.1556/2054.2019.015

Lekhtman, A. (2019, August 23). The first-ever MDMA therapy training for communities of color was vital. Filter. Retrieved from https://filtermag.org/mdma-therapy-people-color/

Michaels, T. I., Purdon, J., Collins, A., \& Williams, M. T. (2018). Inclusion of people of color in psychedelic-assisted psychotherapy: A review of the literature. BMC Psychiatry, 18(245), 1-9. doi:10.1186/s12888-018-1824-6

Neitzke-Spruill, L. (2019). Race as a component of set and setting: How experiences of race can influence psychedelic experiences. Journal of Psychedelic Studies. Advance online publication. doi:10.1556/2054.2019.022

Noorani, T. (2019). Making psychedelics into medicines: The politics and paradoxes of medicalization. Journal of Psychedelic Studies. Advance online publication. doi:10.1556/2054.2019.018

Peluso, D., Sinclair, E., Labate, B., \& Cavnar, C. (2019). Reflections on crafting an Ayahuasca Community Guide for the Awareness of Sexual Abuse. Journal of Psychedelic Studies. Advance online publication.

Phelps, J. (2017). Developing guidelines and competencies for the training of psychedelic therapists. Journal of Humanistic Psychology, 57(5), 450-487. doi:10.1177/0022167817711304

Pollan, M. (2018). How to change your mind: What the new science of psychedelics teaches us about consciousness, dying, addiction, depression, and transcendence. New York, NY: Penguin Press.

Rosenberg, D. E., Wolbach, A. B., Miner, E. J., \& Isbell, H. (1963). Observations on direct and cross tolerance with LSD and D-amphetamine in man. Psychopharmacologia, 5, 1-15. doi:10.1007/bf00405570

Williams, M. T., Reed, S., \& Aggarwal, R. (2019). Culturallyinformed research design issues in a study for MDMA-assisted psychotherapy for posttraumatic stress disorder. Journal of Psychedelic Studies. Advance online publication. doi:10.1556/ 2054.2019.016

Williams, M. T., Rosen, D. C., \& Kanter, J. W. (2019). Eliminating race-based mental health disparities: Promoting equity and culturally responsive care across settings. Oakland, CA: New Harbinger Books. 